\title{
Single Institution Experience of Thymic Tumor Treatment and Survival in Egypt
}

\author{
Dalia Darwish
}

\begin{abstract}
Thymic tumors are the most common tumors in the anterior mediastinum. Total resection is the main treatment and predictor of longer survival. Adjuvant radiotherapy alone or in combination with chemotherapy is recommended with incomplete excision or advanced disease. Thirty seven patients with thymic tumors were included in this retrospective study from January 2001 till December 2012. They were studied regarding age, sex, performance status, tumor size and invasion, stage, pathology, treatment given, overall and progression free survival. Myasthenia gravis was present in $18.1 \%$ of the patients. Masaoka stage III was diagnosed in $40.5 \%$ of the cases followed by stage II in $24.3 \%$ and the other stages with lower percentages. Pathology type B3 was the most frequent followed by $B 2$ and $B 1$ with percentages of $27,24.3$ and 21.7 respectively. Complete resection was conducted in 11 cases $(29.75 \%)$. Partial resection or debulking was done in $15(40.5 \%)$ and a biopsy was taken in 11 cases $(29.8 \%)$ Adjuvant chemotherapy was given to 14 patients $(37.8 \%)$ and neoadjuvant to 13 $(35.2 \%)$. Adjuvant radiotherapy was given to 17 patients $(46 \%)$ and neoadjuvant to $14(37.8 \%)$. The 5-year overall survival by was $83 \%$ for stage I, $71 \%$ for stage II, $60 \%$ for stage III, and $44 \%$ for stage IV (p=0.0426). Five year progression free survival was $71 \%$ for stage I, $62 \%$ stage II, $42 \%$ stage III, and $37 \%$ for stage IV (p= 0.0532). In conclusion with the rare thymic tumors early stage and complete resection have the highest impact on overall and progression free survival.
\end{abstract}

Keywords: Thymic tumors - treatment - survival - Egypt

Asian Pac J Cancer Prev, 17 (2), 771-774

\section{Introduction}

Thymic tumors are the most common tumors in the anterior mediastinum, though they are rare (Engels and Pfeiffer 2003). They occur in adults 40-70 years and are very rare in children or adolescents. Patients usually present with chest pain, cough and dyspnea and few cases are asymptomatic (Detterbeck and Parsons 2011). Fifteen to thirty percent of thymoma patients have myasthenia gravis. Thymomas can be locally invasive but seldom spread to lymph nodes or distant metastasis. Thymic carcinomas are more aggressive and frequently spread to lymph nodes and distant sites (Lopez et al, 2003; Kondo and Monden, 2003).

Thymic carcinoma and type B3 thymoma, have worse prognosis than types $\mathrm{A}, \mathrm{AB}, \mathrm{B} 1$, and $\mathrm{B} 2$. According to Masaoka staging, stage III and IV disease have worse prognosis and higher rate of recurrence in comparison to stage I and II. Five year survival rate for patients with thymoma stages I-III is $85 \%$ and $65 \%$ for stage IV. For thymic carcinomas 5 year survival ranges from $30-50 \%$ (Weksler et al, 2013).

Total resection is the main treatment and predictor of longer survival. Adjuvant radiotherapy is recommended in incomplete excision or advanced disease. Chemotherapy can be given as adjuvant treatment in incomplete resection of thymoma or thymic carcinoma. However, the role of adjuvant chemotherapy in the treatment of thymoma remains unclear (Detterbeck and Zeeshan, 2013; Usman et al, 2015).

\section{Materials and Methods}

Thirty seven patients with thymic tumors were included in this retrospective study from January 2001 till December 2012 in the center of radiation oncology Cairo University. Patients with incomplete records were not included. Patients were studied regarding age, sex, performance status, tumor size and invasion, stage, pathology, treatment given, overall and progression free survival.

Overall survival was determined as the interval between the date of diagnosis, and the date of death or last follow up. Progression-free survival was determined as the time interval between the last day of therapy, and the date of recurrence or last follow-up.

Patients were diagnosed by biopsy or following complete or partial resection. Two types of chemotherapy 
were given, cyclophosphamide, Adriamycin and cisplatin was given to most of the chemotherapy cases, while cisplatin/etoposide was given to 4 patients 2 with stage III and 2 with stage IVa. Chemotherapy was given as neoadjuvant for debulking prior to surgery or as adjuvant treatment post-operative. Radiation therapy either conformal or conventional was given as neoadjuvant treatment for debulking or as adjuvant treatment for residual tumor at a dose ranging from 45-54 Gy.

\section{Statistical analysis}

Data were statistically described in terms of range, mean \pm standard deviation $( \pm$ SD), frequencies and percentages. Kaplan-Meier survival for different parameters was done. A probability value ( $\mathrm{P}$ value) less than 0.05 was considered statistically significant. All statistical calculations were done using computer programs Microsoft Excel 2003 (Microsoft Corporation, NY, USA) and SPSS (Statistical Package for the Social Sciences; SPSS Inc., Chicago, IL, USA) version 15 for Microsoft Windows.

\section{Results}

Thirty seven thymic tumor patients were enrolled in this study from January 2001 till December 2012. Patient's age ranged from 34-61 with a mean of 45.3. Twenty two patients were males $(59.5 \%)$ and fifteen were females $(40.5 \%)$. Dyspnea was the main complaint in most of the cases $(60 \%)$ followed by chest pain in $30 \%$ of the cases with ECOG performance status of 1 in $81 \%$ of the cases. Myasthenia gravis was present in $18.1 \%$ of the patients. Masaoka stage III was diagnosed in $40.5 \%$ of the cases followed by stage II in $24.3 \%$ then the other stages with lower percentage. Pathology type B3 was the most frequent one followed by B2 and B1 with percentages of $27,24.3$ and 21.7 respectively. Patients characteristics is shown in Table1.

Treatment according to stage is shown in Table 2. For stage I all patients underwent complete resection and one patient received adjuvant conformal radiotherapy at a dose of $45 \mathrm{~Gy}$. In stage II patients, $66.7 \%$ underwent complete resection and $33.3 \%$ did partial resection. Four patients $(44.4 \%)$ received adjuvant chemotherapy. Six patients $(66.7 \%)$ received adjuvant radiation therapy 1 received conventional radiotherapy and 5 received conformal radiotherapy. Stage III patients $53.4 \%$ had partial resection, $33.3 \%$ had biopsy and $13.3 \%$ had complete resection. Neoadjuvant chemotherapy was given in $33.3 \%$ while adjuvant was given in $53.4 \%$. Radiation therapy was given as neoadjuvant treatment in $46.7 \%$ and as adjuvant in $46.7 \%$ (7 patients each), $71.4 \%$ received conformal and $28.6 \%$ received conventional radiotherapy. In stage IVa debulking was done in $57.1 \%$ of the cases

Table 1. Characteristics of the Thirty Seven Patients

\begin{tabular}{|c|c|c|}
\hline Characteristic & Number & Percent \\
\hline \multicolumn{3}{|l|}{ Age } \\
\hline$<55$ & 26 & $70.30 \%$ \\
\hline$\geq 55$ & 11 & $29.70 \%$ \\
\hline \multicolumn{3}{|l|}{ Sex } \\
\hline Male & 22 & $59.50 \%$ \\
\hline Female & 15 & $40.50 \%$ \\
\hline \multicolumn{3}{|l|}{ PS (ECOG) } \\
\hline 0 & 2 & $5.40 \%$ \\
\hline 1 & 30 & $81.10 \%$ \\
\hline 2 & 5 & $13.50 \%$ \\
\hline \multicolumn{3}{|c|}{ Myasthenia gravis } \\
\hline No & 30 & $81.10 \%$ \\
\hline Yes & 7 & $18.90 \%$ \\
\hline \multicolumn{3}{|c|}{ Tumor size $(\mathrm{cm})$} \\
\hline$\leq 6$ & 2 & $5.40 \%$ \\
\hline$>6$ & 35 & $94.60 \%$ \\
\hline \multicolumn{3}{|l|}{ Masaoka stage } \\
\hline I & 3 & $8.10 \%$ \\
\hline II & 9 & $24.30 \%$ \\
\hline III & 15 & $40.50 \%$ \\
\hline Iva & 7 & $19 \%$ \\
\hline Ivb & 3 & $8.10 \%$ \\
\hline \multicolumn{3}{|l|}{ Pathology } \\
\hline Type A & 2 & $5.40 \%$ \\
\hline Type AB & 3 & $8.10 \%$ \\
\hline Type B1 & 8 & $21.70 \%$ \\
\hline Type B2 & 9 & $24.30 \%$ \\
\hline Type B3 & 10 & $27 \%$ \\
\hline Type C & 5 & $13.50 \%$ \\
\hline
\end{tabular}

Table 2. Treatment According to Stage

\begin{tabular}{|c|c|c|c|c|c|}
\hline Treatment & $\begin{array}{c}\text { Stage I } \\
N(3)(\%)\end{array}$ & $\begin{array}{c}\text { Stage II } \\
\text { N (9) (\%) }\end{array}$ & $\begin{array}{c}\text { Stage III } \\
\text { N (15) }(\%)\end{array}$ & $\begin{array}{l}\text { Stage IVa } \\
\text { N (7) (\%) }\end{array}$ & $\begin{array}{l}\text { Stage Ivb } \\
\text { N (3) (\%) }\end{array}$ \\
\hline \multicolumn{6}{|l|}{ Surgery } \\
\hline $\mathrm{CR}$ & $3(100 \%)$ & $6(66.7 \%)$ & $2(13.3 \%)$ & & \\
\hline PR & & $3(33.3 \%)$ & $8(53.4 \%)$ & $4(57.1 \%)$ & \\
\hline Biopsy & & 0 & $5(33.3 \%)$ & $3(42.9 \%)$ & $3(100 \%)$ \\
\hline \multicolumn{6}{|l|}{ Chemotheapy } \\
\hline No & $3(100 \%)$ & $5(55.6 \%)$ & $2(13.3 \%)$ & 0 & 0 \\
\hline Neoadjuvant & & 0 & $5(33.3 \%)$ & $5(71.4 \%)$ & $3(100 \%)$ \\
\hline Adjuvant & & $4(44.4 \%)$ & $8(53.4 \%)$ & $2(28.6)$ & 0 \\
\hline \multicolumn{6}{|l|}{ Radiotherapy } \\
\hline No & $2(66.7 \%)$ & $3(33.3 \%)$ & $1(6.6 \%)$ & 0 & 0 \\
\hline Neoadjuvant & 0 & 0 & $7(46.7 \%)$ & $4(57.2 \%)$ & $3(100 \%)$ \\
\hline Adjuvant & $1(33.3 \%)$ & $6(66.7 \%)$ & $7(46.7 \%)$ & $3(42.8 \%)$ & \\
\hline Conventional RT & & $1(16.7 \%)$ & $4(28.6 \%)$ & $2(28.6 \%)$ & $3(100 \%)$ \\
\hline Conformal RT & $1(100 \%)$ & $5(83.3 \%)$ & $10(71.4 \%)$ & $5(71.4 \%)$ & \\
\hline
\end{tabular}


Table 3. Histologic Subtype and the Relationship with Tumor Invasion

\begin{tabular}{|c|c|c|c|c|c|c|}
\hline $\begin{array}{l}\text { Histologic } \\
\text { subtype }\end{array}$ & $\mathrm{I}$ & II & III & Iva & Ivb & $\begin{array}{c}\text { Cases of } \\
\text { invasion to } \\
\text { neighboring } \\
\text { organ } \\
\text { n }(\%)\end{array}$ \\
\hline A & 1 & 1 & & & & $0 \%$ \\
\hline $\mathrm{AB}$ & & 2 & 1 & & & $1(33.3 \%)$ \\
\hline B1 & 1 & 2 & 3 & 2 & & $5(62.5 \%)$ \\
\hline B2 & 1 & 2 & 4 & 2 & & $7(77.8 \%)$ \\
\hline B3 & & 1 & 5 & 2 & 2 & $9(90 \%)$ \\
\hline $\mathrm{C}$ & & 1 & 2 & 1 & 1 & $5(80 \%)$ \\
\hline
\end{tabular}

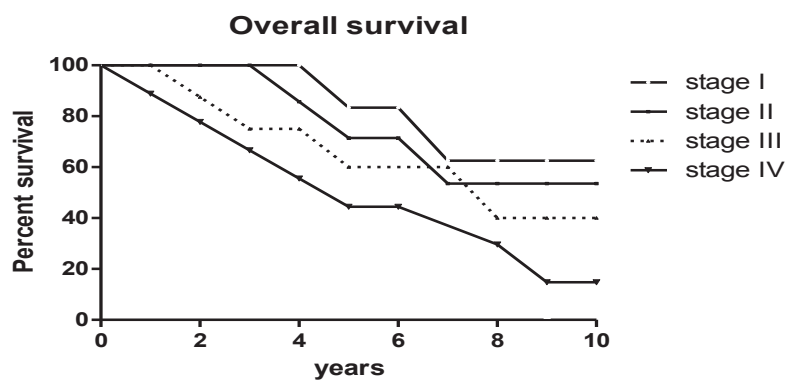

Figure 1. Comparison of Overall Survival for Different Masaoka Stages

PFS

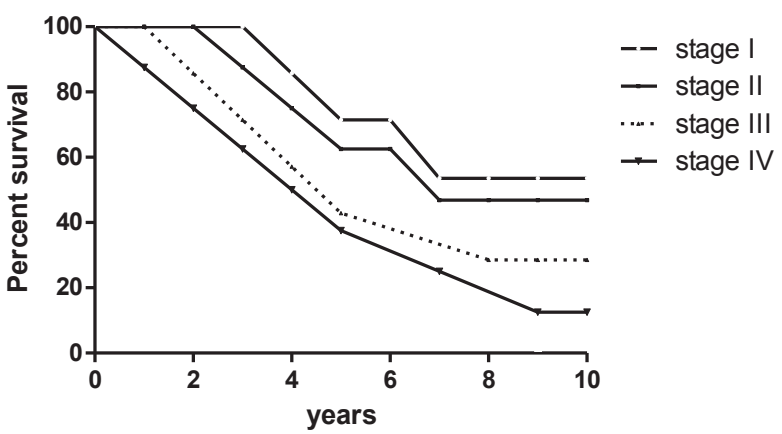

Figure 2. Comparison of Progression free Survival for Different Masaoka Stages

and biopsy was done in $42.9 \%$. Five patients $(71.4 \%)$ received neoadjuvant chemotherapy, while 2 patients (28.6\%) received adjuvant chemotherapy. Neoadjuvant and adjuvant radiotherapy were given in $57.2 \%$ and $42.8 \%$ of the patients respectively, where $28.6 \%$ received conventional and $71.4 \%$ received conformal radiotherapy. For stage IVb the 3 patients did biopsy, received neoadjuvant chemotherapy and neoadjuvant conventional radiotherapy.

Histologic subtype and relationship to tumor invasion showed; type $\mathrm{C}$ had $80 \%$ invasion, $\mathrm{B} 3$ had $90 \%$ invasion to the neighboring organs, type B2 had $77.8 \%$ invasion, type $\mathrm{B} 1$ had $62.5 \%$ invasion, type $\mathrm{AB}$ had $33.3 \%$ invasion, and type A had no invasion the results were not statistically significant table.3.

Five year overall survival for different stages was statistically significant $(\mathrm{p}=0.0426)$. The 5 -year OS by was $83 \%$ for stage I, $71 \%$ for stage II, $60 \%$ for stage III, and $44 \%$ for stage IV figure.1. Five year progression free survival was $71 \%$ for stage I, $62 \%$ stage II, $42 \%$ stage III, and $37 \%$ for stage IV ( $\mathrm{p}=0.0532)$ figure. 2 .

\section{Discussion}

Thymic tumors are the most common tumors in the anterior mediastinum, they occur in adults aged 40-70 years. Patients usually present with chest pain, cough and dyspnea, and myasthenia gravis is present in $15-30 \%$ of the patients (Detterbeck and Parsons 2011; Lopez et al, 2003).

In this study patient's age ranged from 34-61 with a mean of $45.3,59.5 \%$ were males and $40.5 \%$ were females. Dyspnea was present in $60 \%$ of the patients and chest pain in $30 \%$. Myasthenia gravis was present in $18.1 \%$ of the patients.

In our study $8.1 \%$ patients had Masaoka stage I, $24.3 \%$ had stage II, $40.5 \%$ had stage III, and $27.1 \%$ had stage IV, thus stage III had the higher percentage. This was in accordance to the study done by Usman et al, where 5\% of the patients had Masaoka stage I, 17\% had stage II, $45 \%$ had stage III, and $33 \%$ had stage IV disease (Usman et al, 2015).

Complete resection was done in 11 cases $(29.75 \%)$ with stage I, II and 2 with stage III. Partial resection or debulking was done in 15 cases $(40.5 \%)$ and biopsy was done in 11 cases $(29.75 \%)$ stages III and IV. Adjuvant chemotherapy was given in 14 patients $(37.8 \%)$ and neoadjuvant was given in 13 patients $(35.2 \%)$ stages III and IV. Adjuvant radiotherapy was given in 17 patients $(46 \%)$ and neoadjuvant was given in 14 patients stages III and IV (37.8\%).

The 5-year OS by was $83 \%$ for stage I, $71 \%$ for stage II, $60 \%$ for stage III, and $44 \%$ for stage IV ( $p=0.0426)$. Five year progression free survival was $71 \%$ for stage I, $62 \%$ stage II, $42 \%$ stage III, and $37 \%$ for stage IV $(\mathrm{p}=0.0532)$. Thus longer overall and progression free survival was associated with early stages who underwent complete resection with or without adjuvant radiotherapy or chemotherapy.

This was consistent with the previous studies where complete resection has improved survival (Venutaet al, 2010; Chung 2000). In Weksler et al, the median survival for patients who underwent complete excision was 105 months with 5-year survival of 58\%; median survival for patients who did partial excision or biopsy was 29 months with 5-year survival of $26 \%$ (Weksler et al., 2013). Also, the survival of 154 patients with thymic carcinoma done by Kondo and Monden revealed 5-year survivals of $67 \%$ after total resection, $30 \%$ after subtotal resection, and 24\% with biopsy (Kondo and Monden, 2003).

Our results were also comparable to Usman et al results where the 5 -year OS by stage was $80 \%$ for stage I/II, $63 \%$ for stage III, $42 \%$ for stage IVa, and $30 \%$ for stage IVb. The 10 -year OS was $60 \%$ for stage I/II, $42 \%$ for stage III, $28 \%$ for stage IVa, and $13 \%$ for stage IVb (Usman et al, 2015).

In conclusion, thymic tumors are rare tumors usually present late however detection in early stages and complete resection has the highest impact on overall and progression free survival. For advanced stages multimodality treatment is mandatory to improve results. 


\section{References}

Chung D (2000). Thymic carcinoma-analysis of nineteen clinicopathological studies. Thorac Cardiovasc Surg, 48, 114-119.

Detterbeck FC, Parsons AM (2011). Management of stage I and II thymoma. Thorac surg Clin, 21, 59-67.

Detterbeck FC,Zeeshan A (2013). Thymoma: current diagnosis and treatment. Chin Med J Engl, 126, 2186-91.

Engels EA, Pfeiffer RM (2003). Malignant thymoma in the United States demographic patterns in incidence and associations with subsequent malignancies. Int J Cancer, 105, 546-51.

Kondo K, Monden Y (2003). Therapy for thymic epithelial tumors: a clinical study of 1320 patients from Japan. Ann Thorac Surg, 76, 878-84.

López-Cano M, Ponseti-Bosch JM, Espin-Basany E, et al (2003). Clinical and pathologic predictors of outcome in Thymoma-Associated myasthenia gravis. Ann Thoracic Surgery, 76, 1643-9.

Usman A, Xiaopan Y, Frank D et al (2015). Thymic carcinoma outcomes and prognosis: Results of an international analysis. J Thorac Cardiovasc Surg, 149, 95-101.

Venuta F, Anile M, Diso D, et al (2010). Thymoma and thymic carcinoma. Eur J Cardiothorac Surg, 37, 13-25.

Weksler B, Dhupar R, Parikh V, et al (2013). Thymic carcinoma: a multivariate analysis of factors predictive of survival in 290 patients. Ann Thorac Surg, 95, 299-303. 